\title{
聚合物微相分离诱导聚合物功能化碳纳米管的自组装
}

\author{
郑兴良*, 高鸿盛 ${ }^{a, b} \quad$ 丁爱顺 ${ }^{a}$ 李永军*, \\ ( ${ }^{a}$ 长沙理工大学化学与生物工程学院 长沙 410076) \\ $\left({ }^{b}\right.$ 中国科学院上海有机化学研究所 上海 200032)
}

\begin{abstract}
摘要 利用自组装的方法实现碳纳米管的有序排列对制备碳纳米管基复合材料至关重要. 将聚 4-乙烯基吡啶修饰的碳 纳米管与含有羧基的接枝共聚物聚丙烯酸-g-聚甲基丙烯酸甲酷(PAA-g-PMMA)相混合，由于吡啶基团与羧基之间的氢 键作用, 在聚合物微相分离的过程中, 将诱导形成碳纳米管的有序排列, 得到一种基于碳纳米管自组装的蜂窝状膜.

关键词 碳纳米管; 自组装; 微相分离; 复合材料
\end{abstract}

\section{Self-Assembly of Polymer-Functionalized Carbon Nanotubes Induced by Polymer Microphase Separation}

\author{
Zheng, Xingliang ${ }^{*, a} \quad$ Gao, Hongsheng ${ }^{a, b} \quad$ Ding, Aishun ${ }^{a} \quad$ Li, Yongjun ${ }^{*, b}$ \\ ( ${ }^{a}$ Institute of Chemistry and Biological Engineering, Changsha University of Science and Technology, Changsha 410070) \\ ( ${ }^{b}$ Shanghai Institute of Organic Chemistry, Chinese Academy of Sciences, Shanghai 200032)
}

\begin{abstract}
It is of importance for the application of carbon nanotubes in composite materials via supramolecular self-assembly of surface-functionalized carbon nanotubes. Here the self-assembly of the mixture of poly(4-vinylpyridine) functionalized multi-walled carbon nanotubes and carboxyl-containing graft copolymer, poly(acrylic acid)-g-poly(methyl methacrylate) is reported. Because of the hydrogen bond between the pyridine nitrogen and the carboxyl of the graft copolymer, carbon nanotubes were induced to form organized structure during the polymer microphase separation process, which afforded a kind of honeycomb suprastructure containing carbon nanotubes.
\end{abstract}

Keywords carbon nanotube; self-assemble; microphase separation; composite materials

碳纳米管 ${ }^{[1]}$ 由石墨卷曲而成, 碳原子的 4 个价电子 中 3 个可以形成共价键, 每个碳原子贡献 1 个电子形成 离域键, 因此碳纳米管在轴向具有良好的导电性, 其在 光电材料、微电子器件等领域有着很好的应用前景 ${ }^{[2]}$. 为了充分发挥碳纳米管在光电材料和微电子器件方面 等领域的应用，有效地控制碳纳米管的有序排列，利用 自组装的方法实现碳纳米管的有序排列对制备碳纳米 管基复合材料至关重要 ${ }^{[3]}$.

长期以来, 碳纳米管主要是物理学家和材料学家的 研究领域, 化学家仅是将碳纳米管视为一种稳定的无机 材料来研究. 直到 1998 年, 对碳纳米管的表面进行化学 修饰的研究才逐步开展起来. 一般对碳纳米管采用非共 价键以及共价键两种方式进行化学修饰 ${ }^{[4]}$. 非共价键修
饰包括利用有机小分子和高分子与碳纳米管之间的非 共价键的相互作用，例如：静电相互作用、分子间氢键 和 $\pi-\pi$ 相互作用等来完成对碳纳米管的修饰; 共价键修 饰是指利用碳纳米管结构发生特定变化所产生的具有 反应活性的官能团键合上小分子化合物或者是高分子.

一般而言，与小分子修饰相比，高分子修饰后的碳 纳米管不但赋予了其更加优良的溶解性，而且还使得修 饰后的碳纳米管更有利于与其他材料进行杂化, 更有利 于其通过包括静电相互作用、分子间氢键、 $\pi-\pi$ 相互作 用、疏水相互作用等驱动力构建碳纳米管的自组装结 构 $^{[5]}$. Pan 等 ${ }^{[6]}$ 采用机械振动方法, 在空气中将单壁碳纳 米管和氢氧化钾混合震荡反应 $2 \mathrm{~h}$, 得到了表面羟基修 饰的碳纳米管. 由于表面羟基之间的相互作用，单碳纳

\footnotetext{
*E-mail: xingliangzheng@163.com; liyongjun78@sioc.ac.cn

Received January 24, 2013; revised February 20, 2013; published online February 22, 2013.

Project supported by the National Natural Science Foundation of China (No. 20976017) and the 2011 Foundation of Key Laboratory of Organoflurine Chemistry, Shanghai Institute of Organic Chemistry, Chinese Academy of Sciences.

国家自然科学基金(No. 20976017)和中国科学院上海有机化学研究所有机氟化学重点实验室开放基金 2011 资助项目.
} 
米管束平行排列, 组成单碳纳米管的平行阵列. 高超 ${ }^{[7]}$ 制备了聚甲基丙烯酸甲酯修饰的碳纳米管, 发现这种修 饰的多壁碳纳米管可以在金、云母、石英和硅表面上自 组装成一些形态各异的微观结构, 他认为在溶剂挥发过 程中, 高分子链的相分离推动并且稳定了具有刚性的碳 纳米管的自组装. Chen 等 ${ }^{[8]}$ 利用 DNA 链对单碳纳米管 的物理吸附, 将互相配对的 DNA 单链分别对碳纳米管 进行修饰, 并且构建了单壁碳纳米管的自组装, DNA 修 饰的单壁碳纳米管的自组装的推动力来自于互补 DNA 链之间识别和配对作用. Takamori 等 ${ }^{[9]}$ 利用 $\left[\mathrm{N}\left(\mathrm{C}_{12} \mathrm{H}_{25}\right) \mathrm{CH}_{3}\right]^{+} \mathrm{Cl}^{-}$修饰的表面羧酸化的 SWMT, 通过 简单的溶液涂附, 碳纳米管在玻璃片上自组装成蜂窝 状.

分子间的氢键是分子自组装的驱动力之一. 它被广

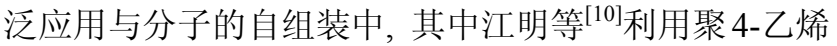
吡啶(P4VP)的吡啶基和含有端羧基的聚丁二烯之间的 氢键成功制备了非共价键键合的胶束. 在本文中, 我们 首先通过表面引发的策略在多壁碳纳米管的表面修饰 上含有吡啶基团的 $\mathrm{P} 4 \mathrm{VP}$, 然后将这种含有吡啶基团的 碳纳米管与含有羧基的接枝共聚物聚丙烯酸- $g$-聚甲基 丙烯酸甲酯(PAA- $g$-PMMA)相混合, 由于吡啶与羧基之 间能够形成氢键, 因此当聚合物发生微相分离时, 将诱 导形成以碳纳米管为基质的自组装结构. 并且研究了聚 合物与碳纳米管的比例、溶剂的体积等因素对碳纳米管 自组装结构的影响. 由于碳纳米管的引入, 将大大提高 自组装结构的力学性能, 而且还可以利用结构规整的蜂 窝状膜为模板构建碳纳米管的有序排列.

\section{1 结果与讨论}

\subsection{P4VP 表面修饰碳纳米管的合成}

与 DNA 相比, 聚合物的很多结构参数具有良好的 可调性，比如聚合物的单体的种类、聚合物的拓扑结构、 聚合物的分子量、聚合物的酸碱性等. 与小分子修饰的 碳纳米管相比, 聚合物修饰的碳纳米管具有更高的韧性 以及更好的延展性. 我们利用硝酸将多壁碳纳米管表面 氧化, 使其表面羧酸化, 然后接入 ATRP 引发基团, 接 着引发 4-乙烯基吡啶聚合得到 P4VP 修饰的碳纳米管 (MWNT-P4VP) (Scheme 1) ${ }^{[11]}$.

通过热重分析验证了上述反应过程的发生. 碳纳米 管具有良好的热稳定性, 在碳纳米管表面进行化学修饰 后，500 ${ }^{\circ} \mathrm{C}$ 以下的热失重主要是有机分子的热分解所引 起的, 而 $500{ }^{\circ} \mathrm{C}$ 以上的热失重主要则是由于碳纳米管 的热分解，因此通过分析反应前后碳纳米管在 $500^{\circ} \mathrm{C}$ 以 下的热失重, 可以验证碳纳米管表面接入了聚合物. 图 1 是碳纳米管表面接入聚合物前后的热失重曲线. 从图
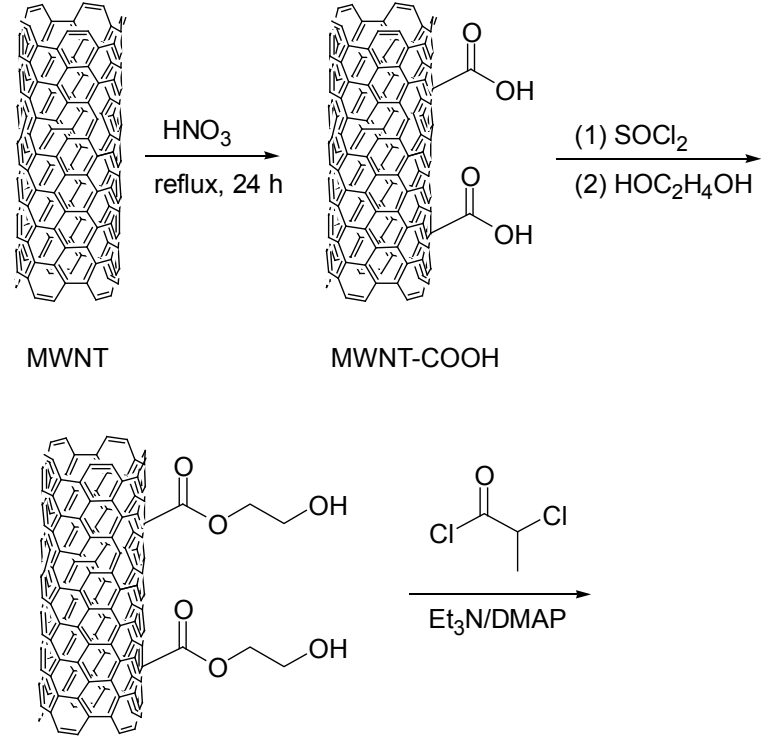

MWNT-OH

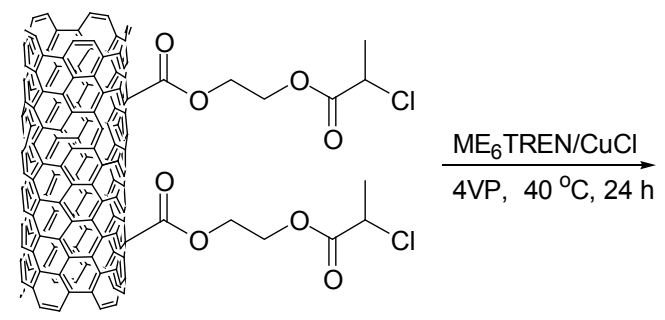

MWNT-Cl

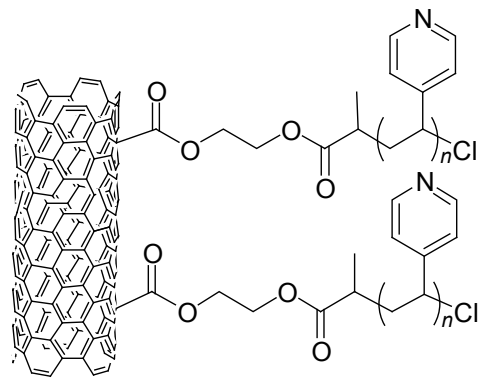

MWNT-P4VP

Scheme 1

中可以看出, 碳纳米管在接入聚合物后在 $500{ }^{\circ} \mathrm{C}$ 以下 有明显的热失重现象, 并且随着有机物含量的增加其热 失重明显增大. P4VP 的含量可以通过 MWNT-P4VP 与 MWNT-Cl 在 $500{ }^{\circ} \mathrm{C}$ 以下失重的差值得到, MWNT$\mathrm{P} 4 \mathrm{VP}$ 中 $\mathrm{P} 4 \mathrm{VP}$ 的含量为 $64.2 \%$.

\subsection{PAA- $b-P M M A$ 的自组装}

两亲性共聚物可以自组装成形态各异的微观结构, 其中一些具有特定结构和性质的聚合物比如线型和星 型的聚苯乙烯、聚丙烯腈和聚苯乙烯的共聚物等可以形 成有序的蜂窝状微孔结构. 我们采用如 Scheme 2 所示 


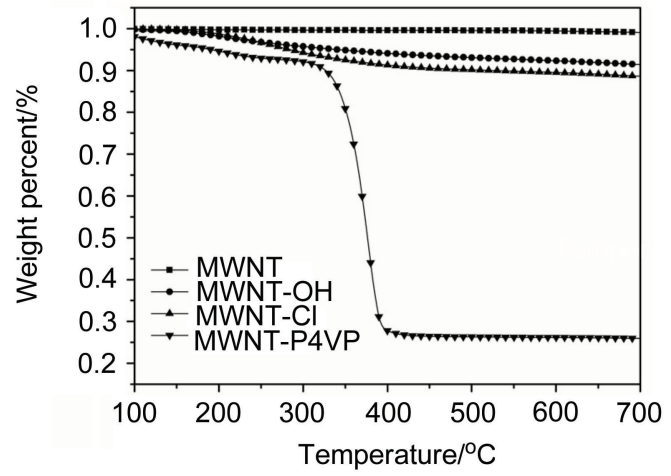

图 1 MWNT, MWNT-OH, MWNT-Cl和 MWNT-P4VP 的热失 重曲线

Figure 1 TGA curves of MWNT, MWNT-OH, MWNT-Cl and MWNT-P4VP

的路线合成了接枝共聚物聚丙烯酸甲氧基甲脂- $g$-聚甲 基丙烯酸甲酯 (PMOMA- $g$-PMMA) $\left(M_{\mathrm{n}}=32.0 \mathrm{kDa}\right.$, $\left.M_{\mathrm{w}} / M_{\mathrm{n}}=1.27, N_{\mathrm{MOMA}} / N_{\mathrm{MMA}}=1 / 21\right)$, 水解后制得 PAA- $g$-PMMA ${ }^{[12]}$.

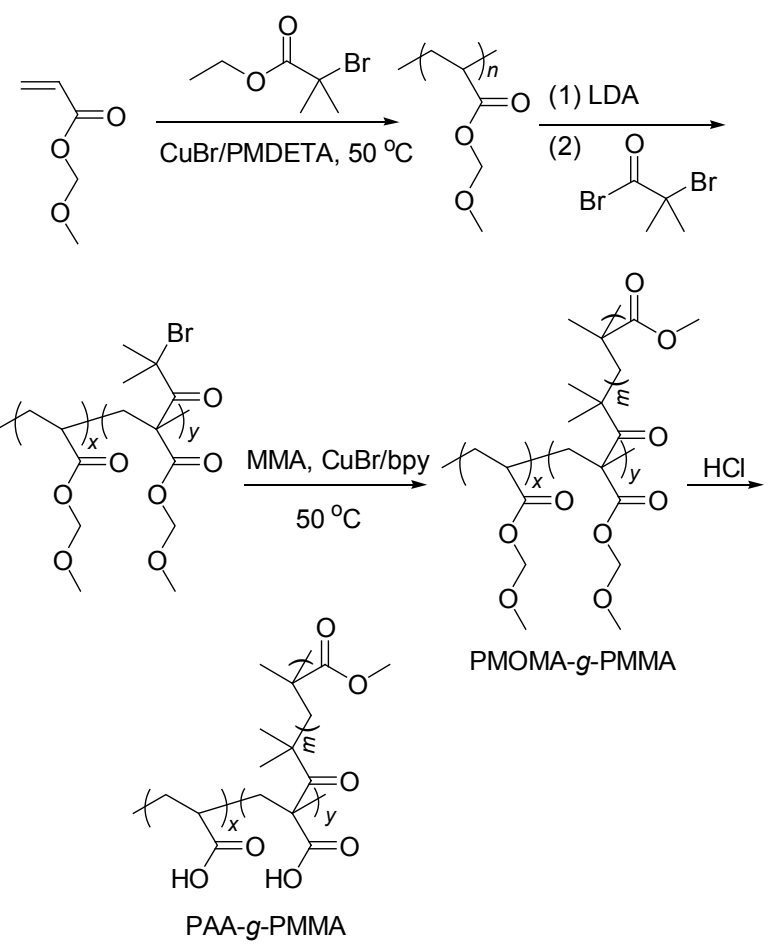

Scheme 2

将 PAA-g-PMMA 配置成不同浓度的氯仿溶液, 旋 涂在云母表面, 然后在室温、 $45 \%$ 相对湿度条件下使溶 剂自然挥发 ${ }^{[13]}$. 由于氯仿的挥发吸收热量从而使得水 汽在聚合物物液膜上冷凝成小水珠, 聚合物就会以这些 小水珠为模板组装成具有多孔结构的膜. 将制备的多孔 结构膜进行 SEM 观察, 从扫描电镜照片上可以发现, 不 同浓度的 PAA- $g$-PMMA 的氯仿溶液可以自组装成结构
规则的蜂窝状结构(图 2). 在 $4 \mathrm{mg} / \mathrm{mL}$ 时, 孔径的分布较 宽，最大的可达 $2 \mu \mathrm{m}$ ，最小的只有 $200 \mathrm{~nm}$ 左右. 浓度减 小后, 其孔径的分布较为均一, 孔径一般都在 $1 \mu \mathrm{m}$ 左 右.

\section{3 聚合物诱导的碳纳米管自组装}

在成功利用 PAA-g-PMMA 的自组装制备蜂窝状的 孔状膜后, 我们将 MWNT-P4VP 与 PAA-g-PMMA 混合, 配置不同浓度的氯仿溶液, 然后在云母上自组装. 由于 碳纳米管引入, 微孔膜的力学性能将大大提高, 而聚合 物的微相分离能够诱导形成碳纳米管的有序排列.

图 3 是 MWNT-P4VP/PAA-g-PMMA 以及 MWNTP4VP 不同浓度的氯仿溶液在云母上自组装的 SEM 图. 不同浓度 MWNT-P4VP/PAA- $g$-PMMA 的氯仿溶液在云 母均形成孔径在 $200 \sim 5 \mu \mathrm{m}$ 不规则的蜂窝状结构, 而不 同浓度的 MWNT-P4VP 在相同的条件下均形成块状结 构. 我们分析产生这种蜂窝结构的过程如下：PAA- $g$ PMMA 与 MWNT-P4VP 同时分散于氯仿中, 在溶剂挥 发过程中, PAA-g-PMMA 将发生微相分离, 由于丙烯酸 与吡啶之间的氢键作用, PAA- $g$-PMMA 链的运动必将 促使 MWNT-P4VP 形成规则的排列，因此 PAA-g-PMMA 的加入能够诱导 MWNT-P4VP 自组装形 态从块状转变为蜂窝状.

\section{2 结论}

我们通过表面引发的方法, 在碳纳米管表面修饰了 含有吡啶基团的聚 4-乙烯基吡啶，将这种修饰过的碳纳 米管与含有羧基的 PAA- $g$-PMMA 相混合，由于羧基与 吡啶基团之间的氢键作用，在聚合物的微相分离过程 中, 诱导碳纳米管排列成有序的结构, 形成多孔蜂窝状 膜. 这种基于超分子作用的碳纳米管自组装结构能够用 于增强多孔膜结构的力学性能, 有望应用于物质分离、 金属及金属氧化物的负载等领域.

\section{3 实验部分}

\section{1 仪器与试剂}

扫描电子显微镜: JEOL 公司 JSM-6390LV 型扫描电 子显微镜. 热失重分析仪: TA 公司热失重分析仪, 在 10 ${ }^{\circ} \mathrm{C} / \mathrm{min}, \mathrm{N}_{2}$ 气流 $20 \mathrm{~mL} / \mathrm{min}$. 凝胶色谱法测定聚合物分 子量及分子量分布: 实验仪器为 Waters 1515 凝胶色谱 仪(HR3, HR4, HR5), 采用 Waters 2410 示差折光检测器 和 Waters 2487 紫外检测器. 用四氢呋喃将聚合物配成 1 $\mathrm{mg} / \mathrm{mL}$ 的溶液, 淋洗剂为四氢呋喃, 流速为 $1 \mathrm{~mL} / \mathrm{min}$, 采用线性聚苯乙烯标样对分子量进行校正.

三乙胺先经 $\mathrm{KOH}$ 干燥数天, 在 $\mathrm{CaH}_{2}$ 存在下回流蒸 

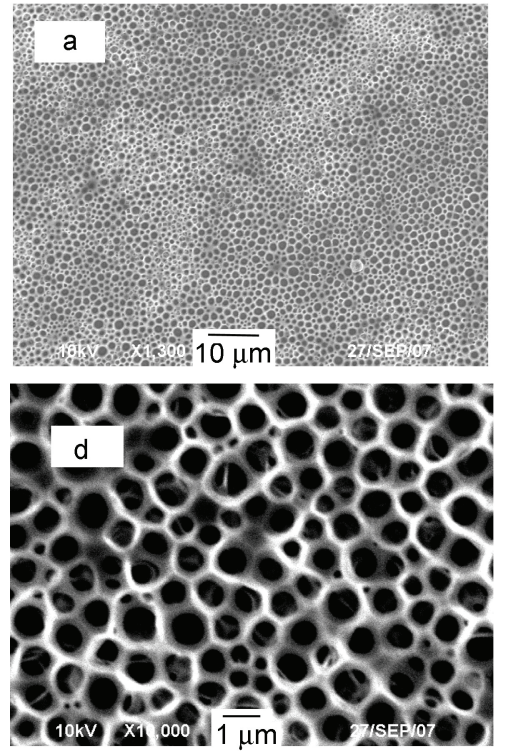
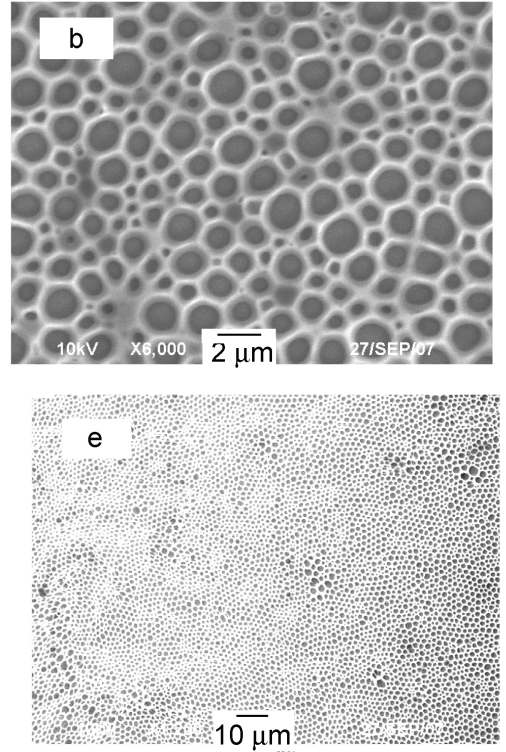
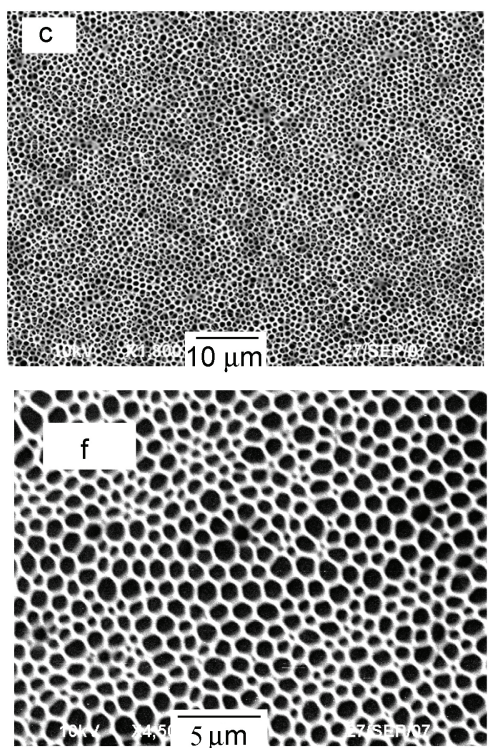

图 2 PAA-g-PMMA 形成的蜂窝状结构膜的 SEM 图

Figure 2 SEM images of PAA-g-PMMA the honeycomb structure of films a, b: $4 \mathrm{mg} / \mathrm{mL}$; c, d: $2 \mathrm{mg} / \mathrm{mL}$; e, f: $1 \mathrm{mg} / \mathrm{mL}$
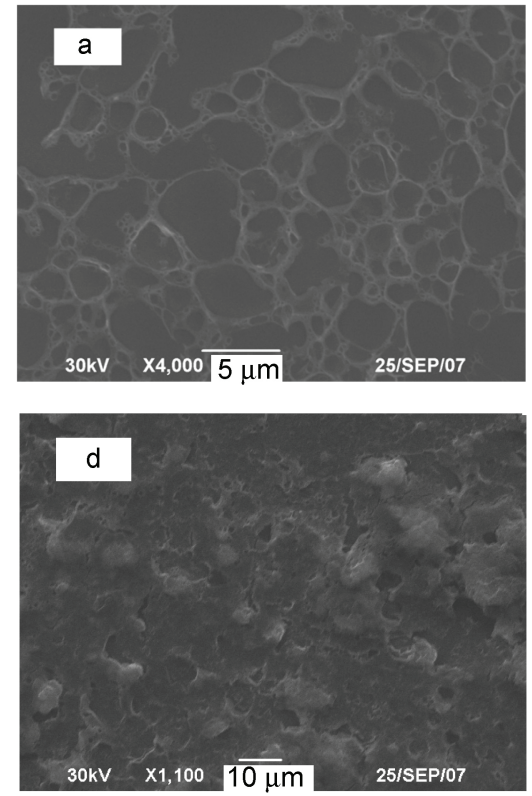

图 3 MWNT-P4VP/PAA-g-PMMA (a, c, e) 以及 MWNT-P4VP (b, d, e)在云母上自组装的 SEM 图

Figure 3 SEM images of PAA-g-PMMA/MWNT-P4VP (a, c, e) and MWNT-P4VP (b, d, e) films a, b: $4 \mathrm{mg} / \mathrm{mL}$; c, d: $2 \mathrm{mg} / \mathrm{mL}$; e, f: $1 \mathrm{mg} / \mathrm{mL}$
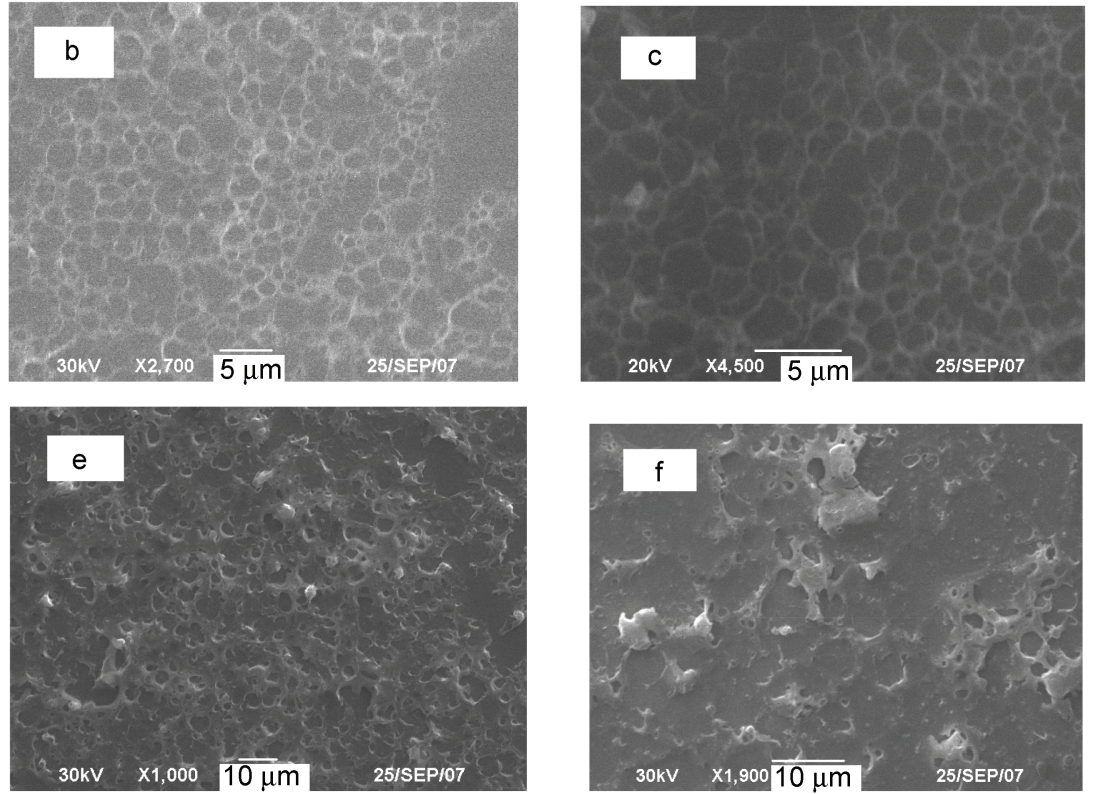

出, 现蒸现用. 溴化亚铜 $(\mathrm{CuCl})$ 经乙酸摚拌过夜后, 再 经乙醇, 乙醚, 丙酮等顺次洗涤数次至淡黄色, 真空烘 箱 $40{ }^{\circ} \mathrm{C}$ 减压干燥 $24 \mathrm{~h}$, 避光保存备用. 甲基丙烯酸甲 酯和 4-乙烯基吡啶均是减压蒸馏两次密封冷冻备用. 其 他未注明试剂均未经处理直接使用.

\section{2 实验方法}

\subsubsection{MWNT-OH 的制备}

向 $50 \mathrm{~mL}$ 蛋形瓶中加入 $2.4592 \mathrm{~g}$ 碳纳米管, $25 \mathrm{~mL}$
浓硝酸, 超声 $30 \mathrm{~min}$ 后加热回流 $24 \mathrm{~h}$, 过滤, 水洗至中 性. $60{ }^{\circ} \mathrm{C}$ 真空干燥 $48 \mathrm{~h}$ 后得 $2.2178 \mathrm{~g}$ 黑色粉末. 将 $1.5026 \mathrm{~g}$ 酸化过的碳纳米管加入到 $50 \mathrm{~mL}$ 蛋形瓶中, 再 加入 $20 \mathrm{~mL} \mathrm{SOCl}$, 超声 $10 \mathrm{~min}$ 后, 置于 $65{ }^{\circ} \mathrm{C}$ 油浴中, 反应 $24 \mathrm{~h}$ 后过滤, 反复用 THF 溶解过滤循环 2 次, 油洜 抽 $0.5 \mathrm{~h}$ 后, 加入到装有 $20 \mathrm{~mL}$ 乙二醇的 $50 \mathrm{~mL}$ 蛋形瓶 中, 置于 $120{ }^{\circ} \mathrm{C}$ 油浴中, 反应 $48 \mathrm{~h}$. 用 THF 反复溶解过 滤循环 4 次, $40{ }^{\circ} \mathrm{C}$ 真空干燥 $48 \mathrm{~h}$ 后得 $1.1408 \mathrm{~g}$ 黑色粉末. 


\subsubsection{MWNT-Cl 的制备}

把装有滴液漏斗的 $50 \mathrm{~mL}$ 三颈瓶, 火烤干燥 2 次除 水, 在氮气保护下加入 $0.4992 \mathrm{~g} \mathrm{MWNT-OH,} 15 \mathrm{~mL}$ $\mathrm{CH}_{2} \mathrm{Cl}_{2}, 0.0438 \mathrm{~g}$ DMAP (3.6 mmol), $0.92 \mathrm{~mL}$ 三乙胺(6.6 $\mathrm{mmol}$ )冰浴 $15 \mathrm{~min}$ 后, 往体系内缓慢滴加 $0.35 \mathrm{~mL} \alpha$-氯 丙酰氯(3.5 mmol)溶于 $5 \mathrm{~mL} \mathrm{CH}_{2} \mathrm{Cl}_{2}$ 的溶液. 反应 $48 \mathrm{~h}$ 后用 $\mathrm{THF}$ 反复溶解过滤 4 次, $40{ }^{\circ} \mathrm{C}$ 真空干燥 $48 \mathrm{~h}$ 后得 $0.3773 \mathrm{~g}$ 黑色粉末. 元素分析(\%): Cl 1.40.

\subsubsection{MWNT-P4VP 的制备}

向干燥的预置有磁子的 Schlenk 瓶中加入 $50.0 \mathrm{mg}$ MWNT-Cl (0.002 mmol Cl), $4.0 \mathrm{mg} \mathrm{CuCl}$ (0.004 mmol), $11 \mu \mathrm{L} \mathrm{Me} \mathrm{M}_{6} \mathrm{TREN}$ (0.004 mmol), $0.09 \mathrm{~mL}$ 4VP (0.08 $\mathrm{mmol}), 0.5 \mathrm{~mL}$ 异丙醇, 经 3 次冷冻-抽真空-溶解通氮 气循环来除去反应液中的氧气后, 超声 $5 \mathrm{~min}$ 后置于 40 ${ }^{\circ} \mathrm{C}$ 油浴中, 反应 $24 \mathrm{~h}$ 后, 用氯仿反复溶解过滤 4 次, 40 ${ }^{\circ} \mathrm{C}$ 真空干燥 $48 \mathrm{~h}$ 后得黑色粉末 $20 \mathrm{mg}$.

\section{2 .4 样品形貌的观察}

$\mathrm{SEM}$ 样品的制备: 在室温下, 将 $30 \mu \mathrm{L}$ 要观察的样 品溶液滴加到云母上, 以 $1500 \mathrm{r} / \mathrm{min}$ 旋涂 $30 \mathrm{~s}$ 后, 室温 干燥后, 室温下真空干燥过夜. 喷金后, 在 JSM-6390LV 型扫描电子显微镜下观察.

\section{References}

[1] Iijima, S. Nature 1991, 354, 56.

[2] (a) Wong, E. W.; Sheehan, P. E.; Lieber, C. M. Science 1997, 277, 1971.

(b) Jia, N. Q.; Lian, Q.; Shen, H. B. Nano Lett. 2007, 7, 2976.

(c) Bianco, A.; Kostarelos, K.; Partidos, C. D. Chem. Commun. 2005, 571.

[3] Park, H.; Afzali, A.; Han, S. J.; Tulevski, G. S.; Franklin, A. D.; Tersoff, J.; Hannon, J. B.; Haensch, W. Nat. Nanotechnol. 2012, 7, 787.
[4] (a) Liu, Z.; Sun, X. M.; Nakayama-Ratchford, N.; Dai, H. J. ACS Nano 2007, 1, 50.

(b) Kong, H.; Gao, C.; Yan, D. Y. J. Am. Chem. Soc. 2004, 126, 412 .

(c) Guo, Z.; Ren, D. M.; Zheng, J. Y. Chin. J. Org. Chem. 2010, 30, 1101 (in Chinese).

(郭震, 任冬梅, 郑健禺, 有机化学, 2010, 30, 1101.)

[5] (a) Yang, D.; Guo, G. Q.; Hu, J. H.; Wang, C.; Jiang, D. L. J. Mater. Chem. 2008, 18, 350.

(b) Yang, D.; Yang, F.; Hu, J. H.; Long, J.; Wang, C. C.; Fu, D. L.; Ni, Q. X. Chem. Commun. 2009, 4447.

(c) Yang, D.; Hu, J. H.; Wang, C. C. Carbon 2006, 44, 3161.

(d) Yang, D.; Zhang, X. H.; Wang, C. C.; Tang, Y. T.; Li, J. J.; Hu, J. H. Prog. Nat. Sci. 2009, 19, 991.

[6] Pan, H. L.; Liu, L. Q.; Guo, Z. X.; Dai, L. M.; Zhang, F. S.; Zhu, D. B.; Czerw, R.; Carroll, D. L. Nano Lett. 2003, 3, 29.

[7] Gao, C. Macromol. Rapid Commun. 2006, 27, 841.

[8] Chen, Y.; Liu, H. P.; Ye, T.; Kim, J. K.; Mao, C. D. J. Am. Chem. Soc. 2007, 128, 8696.

[9] Takamori, H.; Fujigaya, T.; Yamaguchi, Y.; Nakashima, N. Adv. Mater. 2007, 19, 2535.

[10] Chen, D. Y.; Jiang, M. Acc. Chem. Res. 2005, 38, 499.

[11] Sun, Q. W.; Yu, Y.; Zhang, N.; Zhang, F. Y. Chin. J. Org. Chem. 2012, 32, 889 (in Chinese).

(孙庆文, 于颖, 张南, 张法永, 有机化学, 2012, 32, 889.)

[12] (a) Peng, D.; Zhang, X. H.; Feng, C.; Lu, G. L.; Zhang, S.; Huang, X. Y. Polymer 2007, 48, 5250.

(b) Huang, X. W.; Gu, L. N.; Lu, G. L.; Huang, X.; Zhang, Y. Q.; Huang, X. Y. Acta Chim. Sinica 2008, 66, 2653 (in Chinese). (黄晓炜, 顾丽娜, 陆国林, 黄啸, 张亚琴, 黄晓宇, 化学学报, 2008, 66, 2653.)

(c) Huang, X. J.; Xiao, Y.; Lang, M. D. Macromol. Res. 2011, 19, 113.

(d) Huang, X. J.; Xiao, Y.; Lang, M. D. Macromol. Res. 2012, 20, 597.

[13] (a) Ishizu, K.; Makino, M.; Uchida, S. Macromol. Rapid Commun. 2007, 28, 882 .

(b) Lan, P.; Li, J.; G. J. L.; Li, L. Acta Chim. Sinica 2012, 70, 45 (in Chinese).

(兰平, 李剑, 龚剑亮, 李硈, 化学学报, 2012, 70, 45.)

(Qin, X.) 